\title{
Biofriendly micro/nanomotors operating on biocatalysis: from natural to biological environments
}

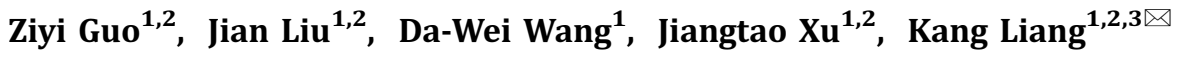 \\ 1 School of Chemical Engineering, University of New South Wales, Sydney, NSW 2052, Australia \\ 2 Australian Centre for NanoMedicine, University of New South Wales, Sydney, NSW 2052, Australia \\ ${ }^{3}$ Graduate School of Biomedical Engineering, University of New South Wales, Sydney, NSW 2052, Australia
}

Received: 22 June 2020 / Accepted: 25 August 2020 / Published online: 4 October 2020

\begin{abstract}
Micro/nanomotors (MNMs) are tiny motorized objects that can autonomously navigate in complex fluidic environments under the influence of an appropriate source of energy. Internal energy-driven MNMs are composed of certain reactive materials that are capable of converting chemical energy from the surroundings into kinetic energy. Recent advances in smart nanomaterials design and processing have endowed the internal energy-driven MNMs with different geometrical designs and various mechanisms of locomotion, with remarkable traveling speed in diverse environments ranging from environmental water to complex body fluids. Among the different design principals, MNM systems that operate from biocatalysis possess biofriendly components, efficient energy conversion, and mild working condition, exhibiting a potential of stepping out of the proof-of-concept phase for addressing many real-life environmental and biotechnological challenges. The biofriendliness of MNMs should not only be considered for in vivo drug delivery but also for environmental remediation and chemical sensing that only environmentally friendly intermediates and degraded products are generated. This review aims to provide an overview of the recent advances in biofriendly MNM design using biocatalysis as the predominant driving force, towards practical applications in biotechnology and environmental technology.
\end{abstract}

Keywords Micro/nanomotors (MNMs), Biofriendly MNM design, Biocatalysis

\section{INTRODUCTION}

Inspired by the diverse molecular motors in nature (Cross 1997; Thomas and Thornhill 1998), recent rapid explosion of materials research and nanotechnology allowed us to explore the potential of artificial micro/nanomotor (MNM) systems for numerous applications (Fernández-Medina et al. 2020). MNM systems present unique properties including controllable motion (Arque et al. 2019), high cargo loading efficiency (Ma et al. 2017), strong towing force (Sanchez et al. 2010), and ease of surface functionalization (Restrepo-Perez et al. 2014). Accordingly, multifarious demonstrations

$\bowtie$ Correspondence: kang.liang@unsw.edu.au (K. Liang) and applications have been developed by exploiting their material tunability (Jurado-Sánchez et al. 2017; Ning et al. 2018; Wang et al. 2016), sensing capabilities (Jurado-Sanchez 2018; Jurado-Sánchez and Escarpa 2017) and controllability (Eskandarloo et al. 2017) for cargo transportation (Ma et al. 2015), environmental remediation (Jurado-Sánchez and Wang 2018; Vilela et al. 2016; Ying et al. 2019) and drug delivery (Guo et al. 2019). A significant amount of MNMs reported to date have focused on the motion manipulation mechanism with potentially environmentally hazardous metalbased catalysts regardless of the manufacturing cost or the naturally feasible working conditions, posing difficulties in the real-world applications of MNMs both in vivo and in the natural environment. Accordingly, 
biocatalysts in general possess higher catalytic efficiency in mild working conditions, endowing biocatalytic MNMs with low cost and high energy efficiency. The great biocompatibility of the MNMs systems is expected to play an essential role in the development of stepping out of the proof-of-concept phase for addressing many real-life environmental and biotechnological challenges.

Recent advances in biocompatible MNMs research have made them become promising candidates for addressing many bio-related challenges owing to their characteristics. These small MNMs present an advantage in overcoming cellular barriers and improving cellular uptake, which made them good nanocarrier substitutes for drug delivery (Esteban-Fernández de Ávila et al. 2018; Gao et al. 2019; Guo et al. 2019; Wang et al. 2019b). However, not only in nanomedicine, employing MNMs in many other areas, for example, cargo delivery (Sanchez et al. 2010), chemical sensing (Orozco et al. 2013), polluted water treatment (Orozco et al. 2014), and reaction catalysis, requires the MNMs to be biofriendly by generating environmental benign intermediates and degraded products. Although the recent development of MNMs has been extensively documented in several reviews, with the majority being focused on the composition, motion mechanism or applications (Sun et al. 2019), here we provide an overarching perspective on MNMs utilizing biocatalysis as the driving force, with a special focus on their biocompatibility in both biological system and the natural environment. Utilizing biocatalysis as the driving force for MNMs offers unique advantages, apart from the excellent specificity and catalytic efficiency, the inherent biocompatible nature of enzymes allows MNMs to operate in mild, biofriendly environments by generating little or no toxic chemical wastes, placing them as an ideal candidate for addressing unmet challenges in biomedicine and the environment.

In this review, we select the most recent and representative work in this field, from our own work and the work by others, to showcase this rapid emerging field. In the first section, we orient our focus on the recent advances in biofriendly MNM design using biocatalysis as the predominant driving force, including choices of biocatalysts and MNM building materials, morphological and size control, and strategies for biocatalysts incorporation. Next, we highlight the practical applications in biomedical and the environmental technology enabled by biocatalytic MNMs, including motion manipulation, water treatment, chemical sensing, nanomedicine, bioimaging, and biosensing (Fig. 1). In the last section, we provide an overview and outlook of the possible biocompatible designs that could be adapted for future use.

\section{BIOCOMPATIBLE AND BIOCATALYTIC MNMS DESIGN}

\section{Biocatalytic reactions as the driving force}

In general, MNM propulsion mechanisms can be categorized into physical driven propulsion (e.g., interfacial surface tension), chemical fuel consumption (e.g., $\left.\mathrm{H}_{2} \mathrm{O}_{2}\right)$, and external field propulsion, such as electrical field, magnetic field, light, and ultrasound (Tu et al. 2017). The propulsion strategies in MNM design exert significant influence on their systematic biocompatibility and consequently, applications. Biocatalytic reactions using enzymes as the catalysts generally exhibit higher energy utilization efficiency and superior biocompatibility in biofriendly environment owing to their excellent substrate selectivity and product turnover rate in mild operating conditions. Accordingly, enzyme-powered MNMs have been extensively explored, where the enzymatic reaction for gas generation being the most dominant focus (Chen et al. 2019; Ma et al. 2016). Biocatalytic MNMs can be propelled by bubble buoyancy (Kumar et al. 2018), the thrust from the bubble ejection (Gao et al. 2012), and the detachment force from the released bubbles (Wang et al. 2006). Maria and co-workers reported an asymmetric mesoporous silica nanomotor functionalized with single-stranded DNA and catalase (Simmchen et al. 2012). The immobilized enzymes produced oxygen bubbles as the driving force from catalytic decomposition of hydrogen peroxide. With functionalized DNA strand on their surface, the nanomotors were capable of capturing and transporting cargos with noncomplementary single-stranded DNA molecule.

Currently, the types of the biochemical fuels for enzyme-powered MNMs are mostly restricted to $\mathrm{H}_{2} \mathrm{O}_{2}$, urea, glucose, and hydrazine (Gao et al. 2014) operating from one or a combination of enzymes (e.g., catalase, glucose oxidase, and urease). Based on the bio-specificity of the chemical fuels, some of the enzyme-powered MNMs can be applied for chemotaxis-driven targeting transportation (Joseph et al. 2017). Battaglia and co-workers reported an enzyme-propelled nanoscopic swimmer by encapsulating glucose oxidase in polymersomes (Joseph et al. 2017). The nano-swimmer exhibited chemotaxis behavior in response to external glucose concentration gradient, which led to the motion towards higher glucose regions. With the aid of lowdensity lipoprotein receptor-related protein 1 (LRP-1) functionalization, the chemotactic behavior of the 


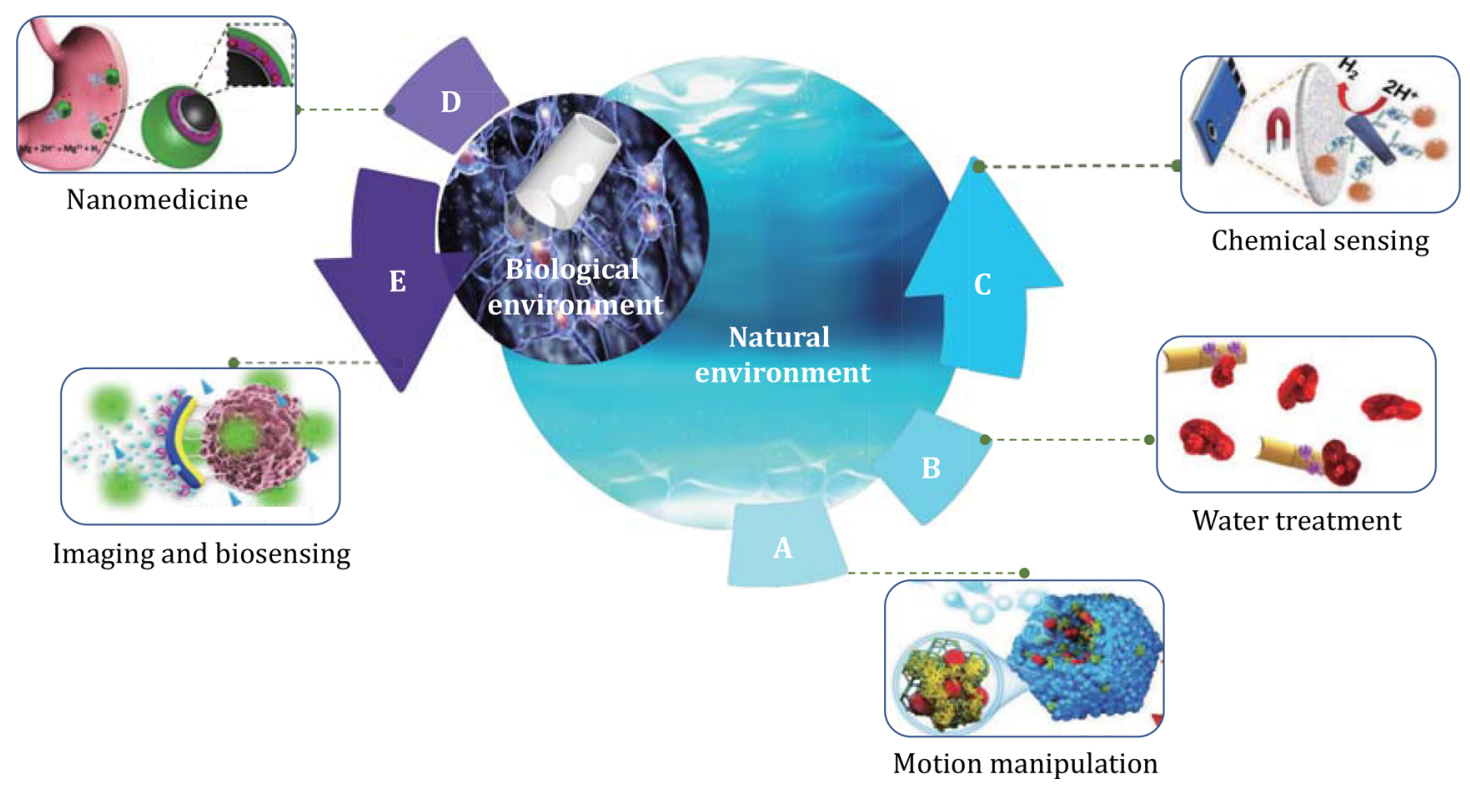

Fig. 1 Overview of biofriendly MNMs driven by biocatalysis for environmental and biotechnological applications. Reproduced with permission: A WILEY-VCH (Gao et al. 2019); B American Chemical Society (Kiristi et al. 2015); C WILEY-VCH (Mayorga-Martinez and Pumera 2019); D Springer Nature (de Ávila et al. 2017); E Elsevier (Zhao et al. 2020)

nanoswimmers demonstrated improved ability in crossing the blood-brain barrier. In another work, different enzymes, including catalase, urease, and ATPase, were coated on liposomes to fabricate biocatalytic motors with positive chemotaxis, negative chemotaxis, and tunable chemotaxis, respectively (Somasundar et al. 2019). The different motion behaviors of the motors were believed to be originated from positive chemotaxis and solute-phospholipid-based negative chemotaxis, as results of different enzyme catalysis routes.

Hitherto, the most popular biofriendly biocatalytic MNMs are propelled by limited types of fuels, which are far less than the traditional catalytic reactions reported for motor propulsion using more toxic inorganic catalysts. Due to the restriction of biocatalytic reaction conditions such as limited $\mathrm{pH}$ and temperature ranges, the utilization of other catalytic reactions to biofriendly systems remained a challenge. To minimize the use of materials hazardous to human health and the environment, the exploitation of more diverse biocatalytic reactions in biological system is expected to be applied as alternatives for biocatalytic MNM design in the future.

\section{Material choices for better biocompatibility}

A variety of materials, including inorganic materials (Coopersmith 2017), organic materials (Wang and Pumera 2017), and hybrid materials (Khezri and Pumera 2019), with diverse chemical compositions have been employed as biocatalytic MNMs matrix. For environmental applications such as water remediation, the toxicity and stability of the material itself are dominant factors for consideration. For biological applications, inappropriate choice of materials could result in inflammation, immunoreaction, or even lethal disease from undesired material-biological interaction (Wang et al. 2019b).

Inorganic materials, including mesoporous silica $\left(\mathrm{SiO}_{2}\right)$ (Llopis-Lorente et al. 2019; Ma and Sánchez 2017), graphene oxide (GO) (Yu et al. 2017), and titanium dioxide $\left(\mathrm{TiO}_{2}\right)$ (Gáspár 2014), are among the most employed materials to construct biofriendly MNMs due to their facile synthetic procedure, adequate stability and ease of surface modification. It is a general consensus that these inorganic materials are abundant in nature with negligible toxicity towards the surrounding environment and living systems at appropriate dosages (Wang et al. 2014).

Besides diverse inorganic materials, synthetic polymers have also been developed to fabricate biofriendly MNMs due to their excellent molecular tunability and facile synthetic procedures, which can equip polymerbased MNMs with proper physicochemical properties, flexible morphologies, diverse functionalities, and nontoxicity (Wong et al. 2016). The diversity of biofriendly polymers imparts significant convenience for the construction of multifunctional MNMs in various aspects from natural environment to biological environment (Somasundar et al. 2019; Wu et al. 2014). For example, 
$\mathrm{Wu}$ and co-workers reported a polyelectrolyte based Janus micromotor system for NIR-light-responsive drug delivery with half coated gold layer (Wu et al. 2014). Biocatalytic enzyme catalase was immobilized on the gold layer to propel the motors using hydrogen peroxide as the fuel in the surrounding environment. The enzymatic motor system exhibited higher catalytic efficiency compared to inorganic Pt-based synthetic motors.

To further extend the compatibility and responsiveness of MNMs in natural and biological environments, hybrid materials that possess desired materials properties from inorganic, organic and even biological components, such as facile synthetic process, the ease of surface functionalization, great porosity and biocompatibility, have been exploited (Khezri and Pumera 2019). Biological components, such as proteins, cell membranes, and even whole cells can also be employed in the fabrication process for MNM surface functionalization and propulsion (Esteban-Fernandez de Avila et al. 2018). Employing hybrid materials in MNM design have tremendous advantages due to their great flexibility and biocompatibility to the surrounding, and they are relatively more sensitive to the specific working environment (Guo et al. 2019). With well-developed fabrication techniques of artificial materials, nano-biohybrid MNMs with greater biocompatibility are expected to be the rising star in MNMs design in future studies.

\section{Morphological and size control}

Recent advances in MNMs research have endowed them various sophisticated shapes and size with great flexibility, which have shown significant impact on their motion patterns and energy conversion efficiency (Patino et al. 2018). With different geometric distribution, the MNMs exhibit structure-dependent motion behavior with various resulting speed and propulsive force. Sánchez and co-workers studied the influence of enzyme distribution and quantity on the motion behavior with urease-conjugated polystyrene (PS) and silicon dioxide-coated polystyrene (PS@SiO ${ }_{2}$ ) micromotors (Patino et al. 2018). Their results demonstrated that the amount of conjugated enzymes was found to be nonlinear to the motion speed and propulsive force resulted from the enzymatic reaction-induced propulsion.

To address biomedical challenges with the aid of MNMs, the size of MNM is one of the most important factors that ultimately determines whether the MNM can effectively penetrate the cell membrane to deliver the loaded therapeutics. It is widely accepted that nanoparticles with diameters ranging from 20 to 200 $\mathrm{nm}$ have advantages in overcoming cellular barriers for drug delivery (Sun et al. 2019). However, most of the existing MNMs systems have a size range at the microscale, which limits their potential for in vivo delivery applications. To circumvent this issue, Wilson and coworkers found that the addition of poly(ethylene glycol) (PEG) was conducive to size and shape control in nanoscale, allowing small polymersomes to transform into stomatocytes (Sun et al. 2019). This ultra-small stomatocyte nanomotor has a diameter around $100 \mathrm{~nm}$ with the capability of encapsulating catalase enzyme as the engine that converts $\mathrm{H}_{2} \mathrm{O}_{2}$ into oxygen bubble to propel the nanomotors. The ability to encapsulate enzymes in the inner compartment of this stomatocyte made it promising nanovesicle candidate for protein delivery and bioimaging. With the small size range and rapid motion, this nanomotor exhibited excellent cell uptake efficiency.

\section{Strategies for biocatalysts incorporation}

Due to the great catalytic efficiency, high substrate specificity, and selectivity, enzymes are widely applied as natural catalysts in various industrial chemical and pharmaceutical production processes. However, being limited by the intrinsic low stability and flexibility, free enzymes showed less environmental tolerance to extreme temperature and $\mathrm{pH}$ (Liang et al. 2015). To enhance the stability, recyclability, and catalytic efficiency of enzymes, many studies have looked into immobilizing enzymes onto various surfaces which exhibited distinct stability improvement without loss of catalytic efficiency (Zhao et al. 2018). The immobilized enzymes on nanoscale support exhibited significantly enhanced mass transfer efficiency and higher diffusion efficiency. For biocatalytic MNMs, strategies for immobilizing enzymes to the motor framework generally include adsorption, encapsulation, and covalent attachment (Wong et al. 2019). Ma and co-workers reported a dual-enzyme-functionalized self-propelled therapeutic nanosystem for synergetic photodynamic therapy (PDT) and starvation therapy (ST) (You et al. 2019). The core NaYF4:Yb,Tm@NaYF4 nanoparticles (UCNPs) and 5,10,15,20-tetrakis(4aminophenyl)porphyrin (TAPP) were encapsulated in ZIF-8 metal-organic framework (MOF) particles through one-pot synthesis, forming UCNPs/TAPP@ZIF-8 (UTZ). To covalently graft the catalase and glucose oxidase enzymes, the UTZ particles were first modified with glutaraldehyde (GA) and then incubated with the enzymes in PBS for $16 \mathrm{~h}$. The $\mathrm{Yb}^{3+}$ in the catalase/GOx functionalized UTZ is capable of harvesting energy from $980 \mathrm{~nm}$ NIR light and transfer to TAPP for ${ }^{3} \mathrm{O}_{2}-{ }^{1} \mathrm{O}_{2}$ transformation. The cascade reaction 
from dual enzymes propelled the motors in solution containing glucose, which greatly enhanced the diffusivity of the micromotors and the cellular uptake efficiency.

In another work, Sánchez and co-workers reported a mesoporous silica nanomotor system with pH-responsive supramolecular nanovalves (Llopis-Lorente et al. 2019). The silica nanomotors were surface-functionalized with benzimidazole group and capped with cyclodextrin-modified urease to prevent the inner cargo from leaking. The urease acted as the biocatalytic engines to propel the motors and the grafted benzimidazole group acted as the valves with the formation of inclusion complexes to release cargo in acidic environments.

Besides covalent attachment, encapsulation is also popular for enzyme immobilization with high loading efficiency. Wilson and co-workers reported an enzymepowered polymeric stomatocytes nanomotor with catalase and glucose oxidase. The stomatocytes were first formed with block copolymer poly(ethylene glycol) ${ }_{44}$ - $b$-poly(styrene) ${ }_{167}$ and the enzymes were mixed with the glassy stomatocytes followed by closing of the stomatocyte neck with solvent addition method. The constructed motors were propelled by the enzymatic reaction and exhibited high speed in biologically relevant fuels. Our group reported a universal and facile strategy to encapsulate almost any kinds of biocatalysts in MOF nanoparticles through a one-step biomineralization process (Liang et al. 2015). Our results demonstrated that enzymes, DNA, proteins, polysaccharides, and even living cells can be encapsulated in MOF particles with high loading efficiency (Liang et al. 2016a, b, 2017) showing great promise in advanced hybrid MNM design for diverse applications.

\section{APPLICATIONS IN BIOMEDICAL AND ENVIRONMENTAL TECHNOLOGY}

\section{Motion manipulation}

Due to the chemical-to-kinetic energy conversion ability, the great mobility against diffusion limit, and continuous stirring of the surrounding environment, MNMs hold great promise for diverse applications from environmental remediation to nanomedicine. Many fundamental studies on MNMs systems are primarily focused on motion and speed manipulation but lack of actual practical applications. However, these fundamental studies shed light to future development in more practical applications employing MNMs. Here, we provide a non-comprehensive highlight of several studies on the enzyme-powered, biofriendly MNMs for motion manipulation towards future practical applications.

The primary design of biocompatible micromotors is simply composed of a micro-engine body and a propelling module. Schmidt and co-workers reported a rolled-up $\mathrm{Ti} / \mathrm{Au}$ microtube with covalently bound catalase on the inner layer (Fig. 2A) (Sanchez et al. 2010). The Au layer was functionalized with self-assembled monolayers (SAMs) of 3-mercaptopropionic acid (3MPA) coupled with 1-ethyl-3[3-dimethylaminopropyl] carbodiimide hydrochloride (EDC) and N-hydroxylsulfosuccinimide (Sulfo-NHS) to form covalent bond between catalase and the inner layer. Catalase allowed oxygen bubble generation from the chemical fuel hydrogen peroxide. As a result, the continuous micromotor motion was achieved by bubble propulsion, which allowed a significant higher carrying force of over $16.44 \mathrm{pN}$ as compared to Pt-based micro-engines.

Multi-enzyme cascade reactions can also be employed in biocompatible enzyme-powered nanomotors. In the study by Sanchez and co-workers, silica nanoparticle template was coated with a mesoporous silica shell and etched with $\mathrm{Na}_{2} \mathrm{CO}_{3}$ to form hollow mesoporous silica nanoparticles (HMSNPs), which were further modified with amino groups and half-capped with a $10 \mathrm{~nm}$ silicon dioxide layer (Fig. 2B) (Ma et al. 2015). The enzymes, including catalase, urease, and glucose oxidase were covalently conjugated to the uncapped side of the HMSNPs to propel the nanomotors with asymmetric biocatalytic reactions using biologically benign fuels such as hydrogen peroxide, urea and glucose. The effective driving force of the biocatalytic nanomotors was measured to be $64 \pm 16 \mathrm{fN}$ with highresolution optical tweezers, which is expected to be further used in directional cargo delivery. In another work, Van Hest and co-workers reported an out-ofequilibrium enzymatic reaction network to propel the micromotors (Nijemeisland et al. 2016). The four cycles of enzymatic reactions enabled the micromotors with tunable and sustained movement using natural glucose and lactate in the body fluids as the fuel.

Apart from direct bubble propulsion, enzyme-powered micromotors can also be activated by bubble-induced buoyancy force. Mann and co-workers reported a catalase-containing organoclay/DNA semipermeable microcapsules with multi-functions including flotation of macroscopic objects, biocatalyst delivery and selfsorting of mixed protocell communities (Kumar et al. 2018). The microcapsules were fabricated by extruding the polyanionic dsDNA (from salmon testes) and catalase with a syringe and dispersing in the exfoliated aminopropyl-functionalized magnesium phyllosilicate (AMP) clay sheets, which gave rise to the 

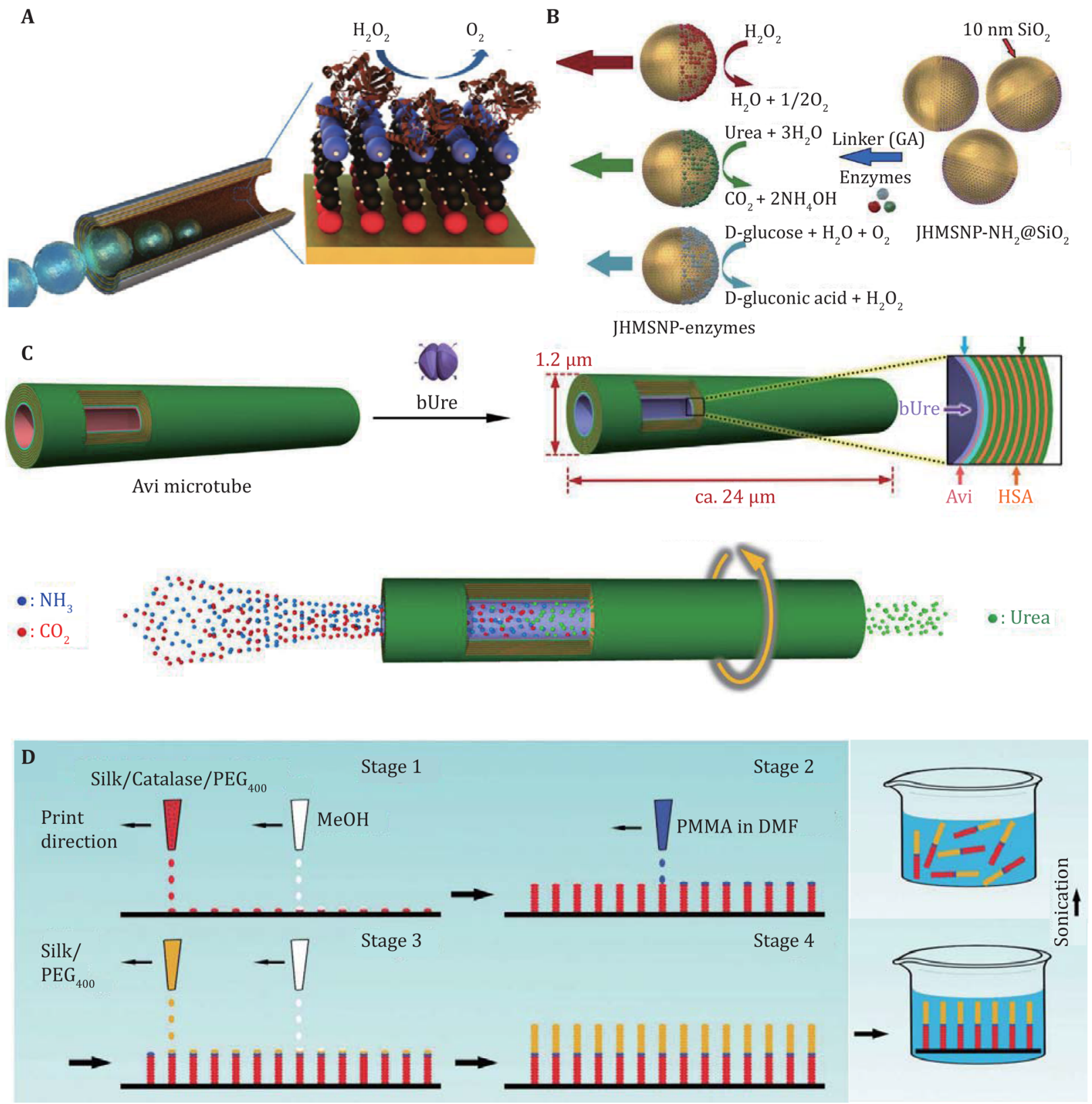

Fig. 2 Micromotor systems for motion control and cargo delivery. A Open view of the hybrid biocatalytic micro-engine with surface modification of inner Au layer and enzymatic decomposition of peroxide fuel. Reproduced with permission of American Chemical Society (Sanchez et al. 2010). B Schematic illustration of enzymatic hollow mesoporous silica Janus nanomotors. Reproduced with permission of Springer Nature (Kumar et al. 2018). C Schematic illustration of preparation of avidin/ biotinylated urease (Avi/bUre) microtube and swimming Avi/bUre microtube with non-bubble propulsion and self-rotation. Reproduced with permission of Wiley-VCH (Sugai et al. 2019). D Schematic of the reactive inkjet printing process for manufacturing biocatalytic micro-rockets. Reproduced with permission of WILEY-VCH (Gregory et al. 2016)

electrostatically induced self-assembly of the microcapsule formation. Co-encapsulation of glucose oxidase to the original microcapsules was exploited to induce sustained oscillatory vertical motion, which decreased the buoyant force by consuming the oxygen microbubbles. The proposed microcapsules exhibited great motility and flexibility for sufficient cargo delivery, which can be further manipulated with remote magnetic guidance.

Apart from the commonly used bubble propulsion to drive enzyme-powered micromotors, a non-bubble propelled protein microtube motor was applied by Komatsu and co-workers in the sodium phosphate buffer solution using urea as the fuel (Fig. 2C) (Sugai 
et al. 2019). The microtubes were fabricated by template synthesis using polycarbonate (PC) membrane with poly(L-arginine) (PLA), human serum albumin and avidin, followed by biotinylated urease coupling onto the internal wall with avidin-biotin interaction. With the diffusion of $\mathrm{NH}_{3}$ and $\mathrm{CO}_{2}$ produced from urea, the microtubes exhibited straight motion with non-bubble propulsion and repetitive lateral self-rotation.

The biocompatible MNMs manufacturing is also applicable using printable materials and printing technology (Fig. 2D) (Gregory et al. 2016). Ebbens and coworkers reported a bubble-propulsive micro-rockets fabricated by inkjet printing by alternating printing of a silk/catalase/polyethylene glycol (PEG) ink and a methanol ink. The proposed reactive inkjet printing was used to alter the distribution of catalase to form asymmetrically propelled micromotors which moved faster in aqueous environments in the presence of hydrogen peroxide fuel. This simple and facile printing technology is expected to open up new potentials in labscale lithographic fabrication processes for miniaturized devices.

\section{Water treatment}

Taking advantage of the mobility, reusability, and ease of collection, self-propelled MNMs are widely investigated in water remediation for heavy metal capture (JuradoSánchez and Wang 2018). However, most of the MNMs proposed for water treatment employ metallic catalysts for propulsion, such as cobalt and platinum (Parmar et al. 2018; Ying et al. 2019), which poses the risk of introducing additional pollutants that is harmful to the environment. Biofriendly materials operating on enzyme catalysis can circumvent this issue by eliminating the need for metallic catalysts and simultaneously exploiting molecules that are innate in the environment as chemical fuels to drive their motion (Wong et al. 2019). Up until now, only a few reports have employed biocatalytic MNM systems in environmental remediation.

The work reported by Wang and co-workers first demonstrated a tubular motor made from commercial pipette tips filled with laccase solution and sodium dodecyl sulfate (SDS) solution (Fig. 3A) (Orozco et al. 2014). This self-propelled microsystem presented a new biocatalytic decontamination strategy by randomly releasing environmental remediation agent while moving. Propelled by the Marangoni effect, the tubular micromotors could navigate in a contaminated solution with effective fluid convection and gradual enzyme release. The well-dispersed enzymatic remediation agent laccase showed great removal efficiency of phenolic pollutant and the motor system was further applied for heavy metal cleanup with substitutive complexing agent EDTA.

In another work from Wang's group, a fully natural biomotor was made of plant tissues cherry-belle radish (Raphanus sativus), which was rich with catalase and peroxidase enzymes (Sattayasamitsathit et al. 2014) (Fig. 3B). Hydrogen peroxide was applied to power the motion of the motors catalyzed by catalase, as well as assisting the transformation of toxic phenolic pollutants as a co-substrate. The motion-induced convection resulted in faster environmental remediation. With additional attachment of catalase to the biomotor, the required peroxide fuel concentration could be dramatically reduced.

\section{Chemical sensing}

Apart from wastewater treatment, the activated motion behavior of biocatalytic MNMs could be used for detecting the presence of aquatic pollutants and assessing the water quality (Jurado-Sánchez and Wang 2018). The great flexibility and mobility of MNMs also endowed the system with great potential for probing solutes in the solutions. Most of the existing MNMs reported were propelled by metallic catalysis including platinum, gold, and magnesium (Jurado-Sánchez and Escarpa 2017; Pacheco et al. 2019). However, biocatalytic MNMs have also been demonstrated as a suitable platform for chemical sensing in aqueous environment.

Wang and co-workers reported a tubular biocompatible microfish using poly(3,4-ethylenedioxythiophene) (PEDOT) microtubes as the backbone and anchoring the catalase enzyme on a mixed self-assembled binary monolayer coupled with EDC/NHS for oxygen bubble propulsion (Fig. 3C) (Orozco et al. 2013). The locomotion and the swimmer survival time were in accordance with the quantity of the contaminants, in response to the impairment of the enzymatic activity. This artificial microfish is expected to offer a real-time sensing capability for water quality by reflecting the presence of heavy metal, pesticide and herbicide.

$\mathrm{Li}$ and the co-workers described an enzyme-powered biodegradable micromotor system for gas sensing applications (Liu et al. 2016) (Fig. 3D). The micromotor was fabricated with polycaprolactone as the backbone. Catalase and fluorescein isothiocyanate (FITC) were immobilized on the motor surface as the biocatalytic engine and signal indicator, respectively. Due to the $\mathrm{pH}$ responsive nature of FITC as a result of fluorescence intensity fluctuation, the micromotors were applied for 

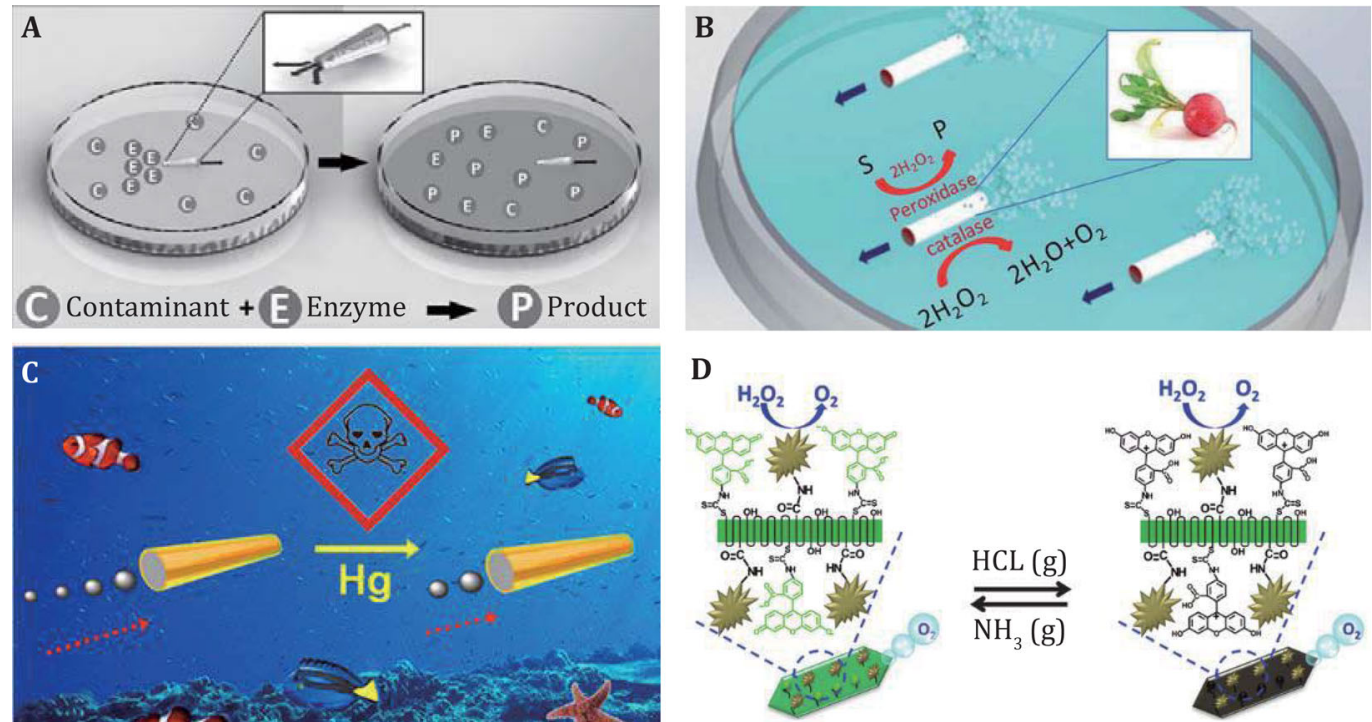

Fig. 3 Micromotor systems working in the natural environment. A Schematic illustration of the concept of motor-based biocatalytic pollutant remediation involving gradual release and mixing of an enzyme. Reproduced with permission of WILEY-VCH (Orozco et al. 2014). B Schematic illustration of the dual-function plant (radish) motors. Reproduced with permission of The Royal Society of Chemistry (Sattayasamitsathit et al. 2014). C Schematic illustration of the pollutant effect on the microfish locomotion speed. Reproduced with permission of American Chemical Society (Orozco et al. 2013). D Schematic representation of self-propelled all-polymer micromotor and the gas sensing behavior Reproduced with permission, The Royal Society of Chemistry (Liu et al. 2016)

acidic or basic gas molecule sensing including hydrogen chloride and ammonia.

\section{Nanomedicine}

A significant number of studies have been reported on the enzyme-powered MNMs for nanomedicine applications (Wang et al. 2019b). Among them, catalase catalyzed bubble propulsion using $\mathrm{H}_{2} \mathrm{O}_{2}$ as the fuel is one of the most practicable mechanisms (Gao et al. 2019; Guo et al. 2019; Wu et al. 2015). Although the excessive amount of the $\mathrm{H}_{2} \mathrm{O}_{2}$ fuel is not biofriendly, the distinct elevated concentration of $\mathrm{H}_{2} \mathrm{O}_{2}$ fuel in tumor and inflamed tissues could sufficiently enhance MNM movement, promoting drug delivery and motion-activated targeting mechanism (Safdar et al. 2018).

Our group developed a MOF-based biocatalytic nanomotor with $\mathrm{pH}$-controlled reversible-speed regulation (Fig. 4A) (Gao et al. 2019). Catalase and succinylated $\beta$-lactoglobulin were encapsulated in nanoporous MOF particles as the engine and gear, respectively. At neutral $\mathrm{pH}$, the $\mathrm{H}_{2} \mathrm{O}_{2}$ fuel could access the catalase through the MOF porous network and produce adequate oxygen bubbles, which could sufficiently propel the MOF nanomotors with thrust. While at mild acidic $\mathrm{pH}$, the $\beta$-lactoglobulin underwent a gelation process, which could block off the MOF pores and hinder the $\mathrm{H}_{2} \mathrm{O}_{2}$ fuel from getting access to catalase, causing diminished nanomotor motion. Results indicated that the cytotoxicity of the doxorubicin-loaded micromotor was originated from two steps. Firstly, drugs were partially released from the motors extracellularly when the accelerated nanomotor motion was triggered in the elevated $\mathrm{H}_{2} \mathrm{O}_{2}$ local environment. Secondly, upon cellular uptake, the MOF-based motor was degraded in the cellular acidic compartments which further released the drugs inside of cell. In another work, we designed a biocatalytic self-propelled submarine-like micromotor with buoyancy controlled directional vertical motion for $\mathrm{pH}$-controlled drug delivery (Fig. 4B) (Guo et al. 2019). The micromotor was constructed with zeolitic imidazolate framework-L (ZIF-L), simultaneously encapsulating bioactive enzyme catalase as the engine and a $\mathrm{pH}$-sensitive polymer poly(2-diisopropylamino)ethyl methacrylate (PDPA) as the switch. In neutral $\mathrm{pH}$ environment, the micromotors could retain the oxygen bubbles produced from catalase at the motor-water interface to produce buoyancy force to efficiently drive the micromotor upward. When it was switched to slightly acidic environments, the PDPA underwent hydrophobic/hydrophilic structural transformation and released the oxygen bubbles, resulting in micromotor downward motion. Consequently the proposed micromotors were capable of targeted delivery of anti-cancer drug 5-FU vertically to the cancer cells at different spatial locations controlled by versatile $\mathrm{pH}$ switches. 


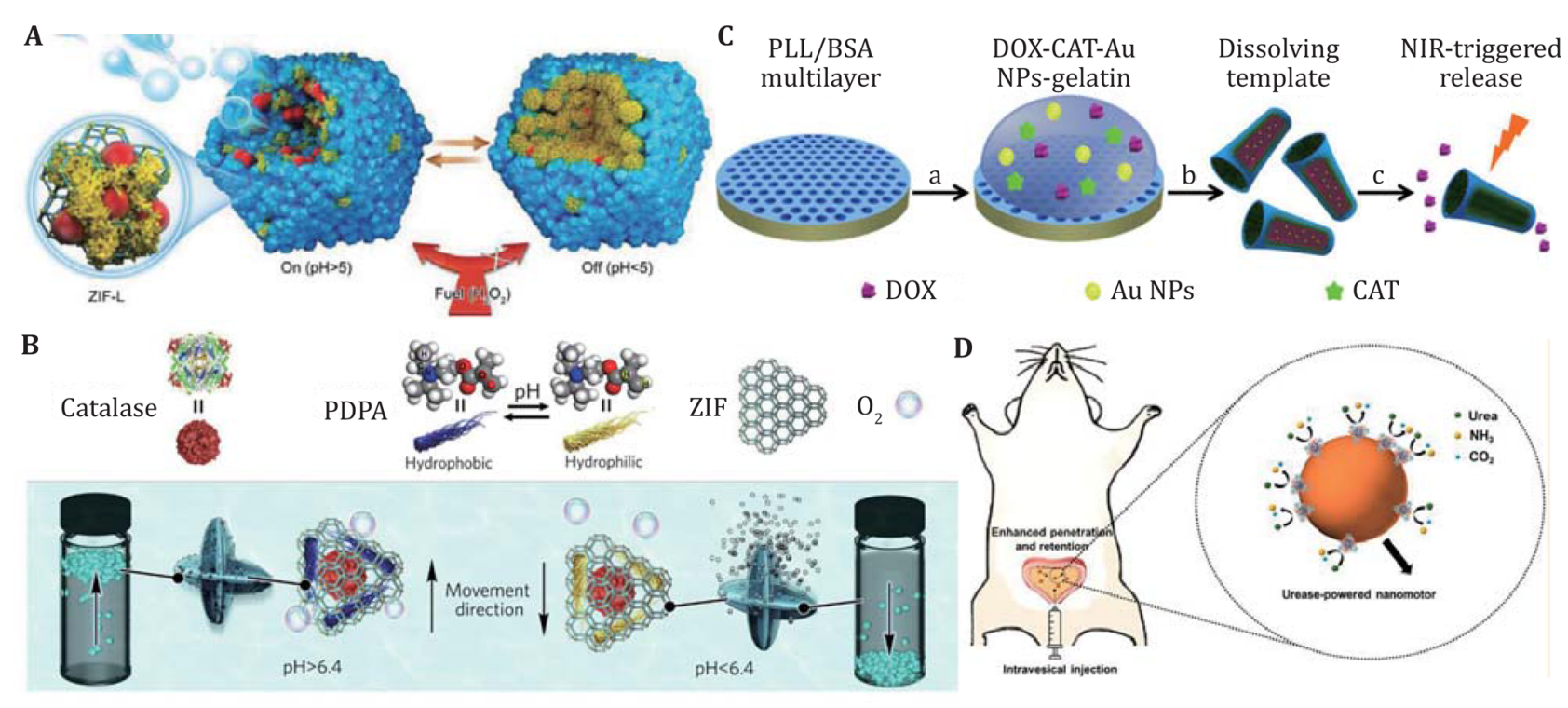

Fig. 4 Micromotor systems for drug delivery. A MOFs-based micromotors with reversible pH-speed regulation. Reproduced with permission of WILEY-VCH (Gao et al. 2019). B Vertical directionally propelled micromotors with pH-controlled hydrophilicity/ hydrophobicity switch. Reproduced with permission of Elsevier (Guo et al. 2019). C PLL/BSA-Based rockets for drug transportation and light-triggered release. Reproduced with permission of American Chemical Society (Wu et al. 2015). D Schematic illustration of intravesical delivery using urease-powered nanomotors. Reproduced with permission of American Chemical Society (Choi et al. 2020)

Considering the drawbacks of toxicity of $\mathrm{H}_{2} \mathrm{O}_{2}$ as chemical fuels, some other studies adapted alternative ways to enhance the motors' biocompatibility by exploiting more biofriendly biomolecules such as glucoses (Schattling et al. 2015) and urea (Dey et al. 2015; Hortelão et al. 2018; Ma et al. 2015) as the fuel. Städler and co-workers developed a glucose-propelled Janus particle system with an in-built enzyme cascade reaction (Schattling et al. 2015). Glucose oxidase (GOx) and catalase were co-immobilized in the particles and the biocompatible fuel glucose was decomposed by GOx to produce $\mathrm{H}_{2} \mathrm{O}_{2}$, followed by the catalytic reaction of catalase to form oxygen bubbles. The cascade reaction resulted in lower power output to the mobile system compared to that of direct usage of $\mathrm{H}_{2} \mathrm{O}_{2}$ fuel and inorganic-based catalyst, but the intermediate product $\mathrm{H}_{2} \mathrm{O}_{2}$ was kept at a safer level for the benefit of the systematic biocompatibility. Considering the biocompatibility of both the motor and the chemical fuel, this system is believed to be suitable for in vivo applications, particularly as self-propelled drug delivery. In a following work, the authors expanded the biocompatible Janus micromotor system to a double-fueled system with glucose and peptides, which enhanced the power of engines without compromising their biocompatibility (Schattling et al. 2017).

The precise controlling of the drug release is also one of the main MNMs research priorities in nanomedicine. To this end, $\mathrm{Wu}$ and co-workers reported a poly-L- lysine/bovine serum albumin (PLL/BSA) multilayer rocket consisted of heat-sensitive gelatin hydrogel, gold nanoparticles, doxorubicin and catalase (Fig. 4C) (Wu et al. 2015). The drugs were delivered to the cancer cells through the biocatalytic bubble propulsion with additional precision by magnetic guidance. When they arrived at the cancer cells, exposing the motors to NIR irradiation could produce heat as a result from the photothermal effects of gold nanoparticles and dissolve gelatin to release doxorubicin to the surrounding cancer cells. With great flexibility, biodegradability and multifunctionality, this micromotor system showed a promising future for in vivo drug delivery.

So far, most of the works involving MNMs for drug delivery were based on in vitro two-dimensional cell culture, while the micro-environments for practical in vivo drug delivery are much more complex. Sanchez and co-workers took a step forward and built a ureasepowered nanomotors with anti-FGFR 3 antibody on the outer surface (Hortelão et al. 2019). The micromotors were propelled with urea and could specifically target the 3D spheroids bladder cancer cells, which was believed to be a closer model to the real tumor in vivo. The proposed nanomotor carrier showed a significantly higher internalization efficiency into the spheroids compared to that of passive particles and presented enhanced cancer therapy effect.

Very recently, Hahn and the co-workers reported a urease-powered nanomotor for intravesical therapy of 
bladder diseases (Choi et al. 2020) (Fig. 4D). The nanomotors were constructed with polydopamine (PDA) nanocapsules on sacrificial silica nanoparticles, which were then etched away with hydrofluoric acid (HF). Urease was attached to the PDA surface through Schiff base reaction between amine groups on urease and catechol groups on PDA. Propelled by the biocatalytic conversion of urea, the nanomotors could penetrate into the bladder tissue and prolong the retention time after repeated urination. This nanomotor system could be used as sustained release drug carriers for bladder diseases.

Wang and co-workers reported an enzyme-powered cell robots with natural platelet cells (Tang et al. 2020). The biocatalytic enzyme urease was grafted asymmetrically on the surface of the bare cells to enable the chemophoretic motion. The surface functionalized platelet cells exhibited intrinsic biofunctionalities of cancer cell targeting, which is sufficient for targeted drug delivery. The hybrid system combined the merits from both biocompatible enzymatic reaction-induced motion and the intrinsic capacity of natural cells, offering inspiring avenues for biocompatible MNMs system development.

Despite the rapid progress, enzyme-powered MNM for nanomedicine is still in the early proof-of-concept phase. Very limited researches have assessed enzymepowered MNM systems in vivo although other kinds of MNMs have been reported for in vivo drug delivery, such as metal catalyst-based MNMs (de Ávila et al. 2017; Esteban-Fernández de Ávila et al. 2018; Li et al. 2017). It may be attributed to the instability of enzymes when they are delivered inside the body, leading to hydrolyzation and degradation by the living organism. In addition, less biofriendly fuel such as $\mathrm{H}_{2} \mathrm{O}_{2}$, needs to be kept at lower level or replaced by other more biofriendly fuels. Nevertheless, with the research of new nanomaterials that can protect enzymes even inside the body (Guo et al. 2020; Liang and Liang 2020), enzymepowered MNMs are expected to open a new era in nanomedicine.

\section{Imaging and biosensing}

Bioimaging techniques are widely accepted as the core of prompting diagnosis, which played an important role in assessing the progress of disease and precise surgery preparation (Sun et al. 2017; Wang et al. 2018). Biomacromolecules and micro-environments of tumor cells are often recognized as the biomarkers of cancer (Felder et al. 2014). In recent years, tremendous attention has been paid to the development of bioimaging enhancement with MNMs (Peng et al. 2017).
Mattery and co-workers reported nanomotor assisted ultrasound imaging in the abscess in rats (Fig. 5A) (Olson et al. 2013). Poly (sodium styrene sulfonate) (PSS) was used as the motor matrix with negativecharged surface, which could be further functionalized for cell targeting, and catalase was immobilized as the biocatalytic engine with local $\mathrm{H}_{2} \mathrm{O}_{2}$. The locally produced microbubbles were considered as specific signals for activated neutrophils in ultrasound imaging with high sensitivity. Based on this mechanism, this micromotor system was able to distinguish activated neutrophils from native ones due to the lack of $\mathrm{H}_{2} \mathrm{O}_{2}$ in native neutrophils. According to the bioimaging results of injected micromotors in the abscess of rats, the groups with activated neutrophils showed an enhanced ultrasound signal compared to the conventional microscopy techniques.

Apart from bioimaging, MNMs assisted biosensing was also well discussed and accepted in disease diagnosis (Jurado-Sánchez and Escarpa 2017; Kong et al. 2018). Some efforts have been made to fulfill the need of early-stage tumor detection with the MNMs (Gao et al. 2018; Wang et al. 2019a). Ju and co-workers recently developed a jellyfish-like micromotor with DNA detecting assembly and biocompatible enzyme catalase, which was decorated on the concave surface of the motor (Fig. 5B) (Zhang et al. 2019). The motor exhibited quick moving behavior in bio-media from the catalytic reaction of catalase in the presence of $\mathrm{H}_{2} \mathrm{O}_{2}$. When the specific DNA sequences were detected and matched by the DNA self-assembly, the micromotor disassembled and resulted in the detachment of the catalase. Therefore, the proposed system was applicable in DNA sensing with the speed of motion corresponding to the presence of the target DNA. This micromotor provided a facile method to detect the biomacromolecules with a good sensitivity and reproducibility.

Sánchez and co-workers reported a urease-powered micromotor with synthetic DNA nano-switch which acted as a photostable FRET-based probe (fluorescence resonance energy transfer) (Fig. 5C) (Patino et al. 2019). This sensor could provide real-time monitoring of micro-environment $\mathrm{pH}$ changes through FRET/Cy3 (cyanine-3 fluorophore) ratio fluctuation in a few seconds. The versatile platform was proved to be applicable in intracellular $\mathrm{pH}$ which is suitable for sensing the tumor micro-environment.

In another work, Zhao and co-workers reported Janus micromotors for circulating tumor cells detection with motion-capture-ratiometric fluorescence changes (Fig. 5D) (Zhao et al. 2020). The micromotor system was propelled by decomposing hydrogen peroxide with catalase, which was grafted on one side of the Janus 

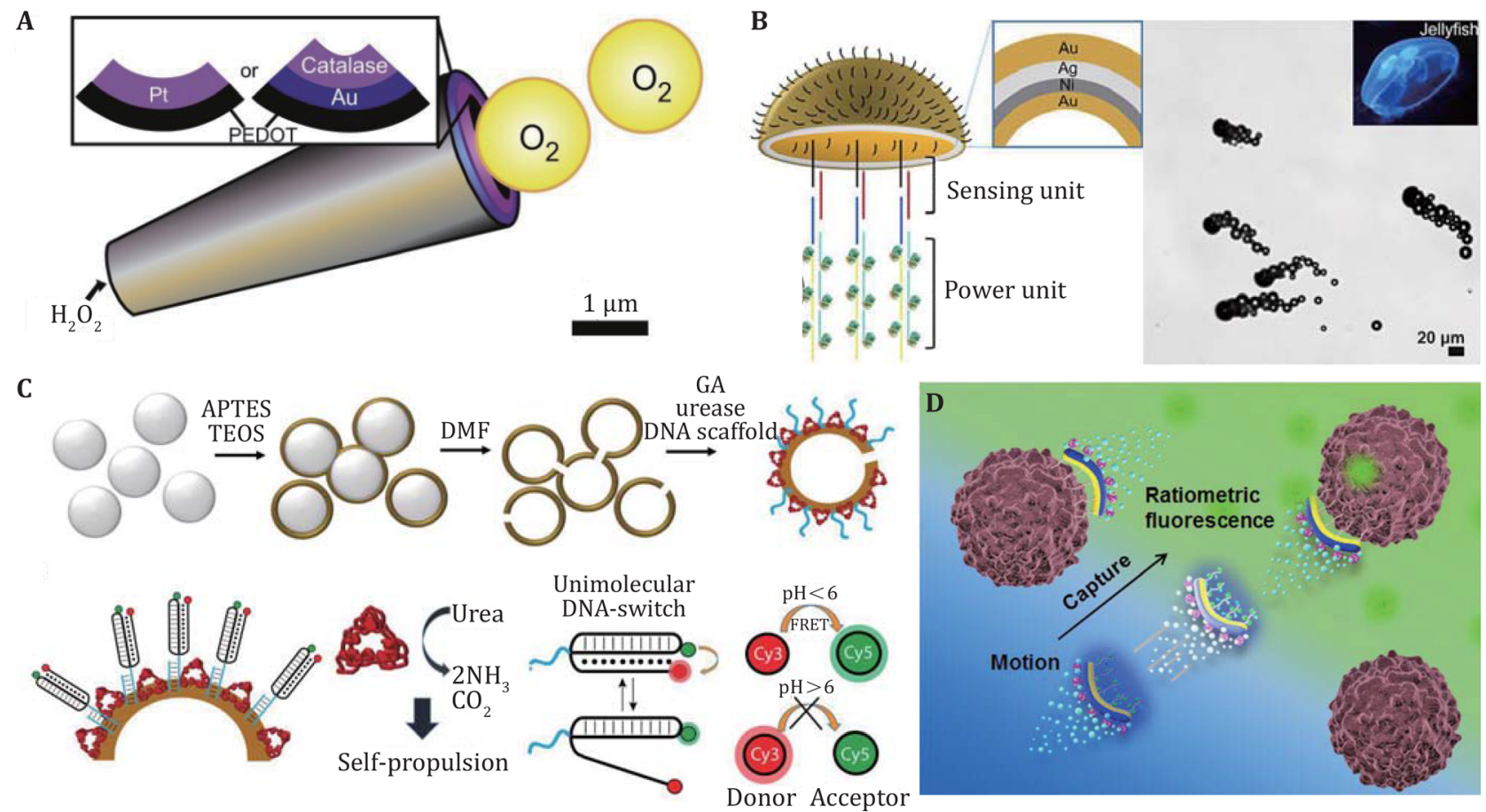

Fig. 5 Micromotor systems for biosensing. A Schematic illustration of ultrasound-based visualization of oxygen microbubbles formed by micromotor converters. Reproduced with permission of Elsevier (Olson et al. 2013). B Schematic illustration of micromotors used for specific DNA sensing. Reproduced with permission of American Chemical Society (Zhang et al. 2019). C Schematic representation of the micromotors fabrication, where a silicon dioxide layer is grown onto a commercial polystyrene template. Reproduced with permission of American Chemical Society (Patino et al. 2019). D Schematic illustration of ratiometric fluorescence response of Janus micromotors after capture of tumor cells. Reproduced with permission of Elsevier (Zhao et al. 2020)

rods, and TLS11a aptamers were decorated on the other side of Janus rods for cell targeting. To achieve fluorescence indication, thymine and guanine were conjugated on tetraphenylethene (TPE) and fluorescein isothiocyanate (FITC) followed by grafting onto aptamers via basepair interactions. Due to the aggregationinduced emission (AIE) of TPE and aggregation-caused quenching of FITC, the competitive binding of tumor cells with aptamers resulted in the fluorescence change from blue to green. This motor system demonstrates the capabilities of motion-capture ratiometric fluorescence detection of tumor cells with high selectivity, rapid recognition, and low detection limit.

\section{CONCLUSION AND PERSPECTIVES}

In this review, we capture the recent research efforts in biocompatible MNMs that operate on enzyme-powered catalysis with different constructions and applications ranging from biomedicine to the environment. The rapid explosion of nanomaterial research in the past two decades has enabled diverse propulsion mechanisms and possible applications. At present, the full promise of MNMs constructs to real-world applications has still not been met as the field has only begun to mature recently. One of the major challenges remained for real-world application is the compatibility of MNMs with the biological system and the natural environment. There are still works to be performed on designing new techniques and strategies for integrating biocompatible MNMs with diverse applications. Another important consideration in engineering these MNMs is the endogenous available energy source or, at least, biofriendly fuels available for their motion. The excessive amount of fuels required for MNM motion could be harmful to the biological system and natural environment. Therefore, it is important to develop more energy efficient motors with less fuel demand. The current biocompatible MNMs generally revolve around enzyme biocatalysis, particularly catalase for decomposing hydrogen peroxide. In contrast, biological system utilizes thousands of different enzymes to catalyze diverse biochemical reactions. This represents new opportunity for MNMs by exploiting new biocatalytic pathways. In addition, the optimization of MNMs properties-such as size, morphology, geometry, and catalyst distributionhas shown great influence on the motion performances, which should play an important role in guiding or enhancing MNMs development. It is anticipated that 
advancements in these areas will lead to an improved ability in designing and optimizing biocompatible MNMs that can be ultimately employed for many applications.

Acknowledgement This work is supported by the Australia National Health and Medical Research Council (NHMRC, APP1163786), the Australian Research Council (ARC, DP190101008), and the Scientia Fellowship program at UNSW. Ziyi Guo thanks the support by the Australian Government Research Training Program Scholarship. Jian Liu acknowledges the University International Postgraduate Scholarship of UNSW Sydney.

\section{Compliance with Ethical Standards}

Conflict of interest Ziyi Guo, Jian Liu, Da-Wei Wang, Jiangtao Xu, and Kang Liang declare that they have no conflicts of interest.

Human and animals rights and informed consent This article does not contain any studies with human or animal subjects performed by the any of the authors.

Open Access This article is licensed under a Creative Commons Attribution 4.0 International License, which permits use, sharing, adaptation, distribution and reproduction in any medium or format, as long as you give appropriate credit to the original author(s) and the source, provide a link to the Creative Commons licence, and indicate if changes were made. The images or other third party material in this article are included in the article's Creative Commons licence, unless indicated otherwise in a credit line to the material. If material is not included in the article's Creative Commons licence and your intended use is not permitted by statutory regulation or exceeds the permitted use, you will need to obtain permission directly from the copyright holder. To view a copy of this licence, visit http://creativecommons.org/ licenses/by/4.0/.

\section{References}

Arque X, Romero-Rivera A, Feixas F, Patino T, Osuna S, Sanchez S (2019) Intrinsic enzymatic properties modulate the selfpropulsion of micromotors. Nat Commun 10(1):2826

Chen C, He Z, Wu J, Zhang X, Xia Q Ju H (2019) Motion of enzymepowered microshell motors. Chem Asian J 14(14):2491-2496

Choi H, Cho SH, Hahn SK (2020) Urease-powered polydopamine nanomotors for intravesical therapy of bladder diseases. ACS Nano 14(6):6683-6692

Coopersmith KJ (2017) Putting nanoparticles to work: Selfpropelled Inorganic Micro- and Nanomotors. In: Hunyadi Murph SE, Larsen GK, Coopersmith KJ et al (eds) Putting nanoparticles to work: self-propelled inorganic micro- and nanomotors. Springer, Cham, pp 153-168

Cross RA (1997) Molecular motors: the natural economy of kinesin. Curr Biol 7(10):R631-R633

de Ávila BE-F, Angsantikul P, Li J, Angel Lopez-Ramirez M, Ramírez-Herrera DE, Thamphiwatana S, Chen C, Delezuk J, Samakapiruk R, Ramez V, Obonyo M, Zhang L, Wang J (2017) Micromotor-enabled active drug delivery for in vivo treatment of stomach infection. Nat Commun 8:272

Dey KK, Zhao X, Tansi BM, Méndez-Ortiz WJ, Córdova-Figueroa UM, Golestanian R, Sen A (2015) Micromotors powered by enzyme catalysis. Nano Lett 15(12):8311-8315
Eskandarloo H, Kierulf A, Abbaspourrad A (2017) Light-harvesting synthetic nano- and micromotors: a review. Nanoscale 9(34):12218-12230

Esteban-Fernandez de Avila B, Gao W, Karshalev E, Zhang L, Wang J (2018a) Cell-Like micromotors. Acc Chem Res 51(9):1901-1910

Esteban-Fernández de Ávila B, Angsantikul P, Li J, Gao W, Zhang L, Wang J (2018b) Micromotors go in vivo: from test tubes to live animals. Adv Funct Mater 28(25):1705640

Felder M, Kapur A, Gonzalez-Bosquet J, Horibata S, Heintz J, Albrecht R, Fass L, Kaur J, Hu K, Shojaei H, Whelan RJ, Patankar MS (2014) MUC16 (CA125): tumor biomarker to cancer therapy, a work in progress. Mol Cancer 13(1):129

Fernández-Medina M, Ramos-Docampo MA, Hovorka O, Salgueiriño V, Städler B (2020) Recent advances in nano- and micromotors. Adv Funct Mater 30(12):1908283

Gao W, Pei A, Wang J (2012) Water-driven micromotors. ACS Nano 6(9):8432-8438

Gao W, Pei A, Dong R, Wang J (2014) Catalytic iridium-based Janus micromotors powered by ultralow levels of chemical fuels. J Am Chem Soc 136(6):2276-2279

Gao W, de Ávila BE-F, Zhang L, Wang J (2018) Targeting and isolation of cancer cells using micro/nanomotors. Adv Drug Deliv Rev 125:94-101

Gao S, Hou J, Zeng J, Richardson JJ, Gu Z, Gao X, Li D, Gao M, Wang D-W, Chen P, Chen V, Liang K, Zhao D, Kong B (2019) Superassembled biocatalytic porous framework micromotors with reversible and sensitive $\mathrm{pH}$-speed regulation at ultralow physiological $\mathrm{H}_{2} \mathrm{O}_{2}$ concentration. Adv Funct Mater 29(18):1808900

Gáspár S (2014) Enzymatically induced motion at nano- and micro-scales. Nanoscale 6(14):7757-7763

Gregory DA, Zhang Y, Smith PJ, Zhao X, Ebbens SJ (2016) Reactive inkjet printing of biocompatible enzyme powered silk microrockets. Small 12(30):4048-4055

Guo Z, Wang T, Rawal A, Hou J, Cao Z, Zhang H, Xu J, Gu Z, Chen V, Liang K (2019) Biocatalytic self-propelled submarine-like metal-organic framework microparticles with pH-triggered buoyancy control for directional vertical motion. Mater Today 28:10-16

Guo Z, Richardson JJ, Kong B, Liang K (2020) Nanobiohybrids: materials approaches for bioaugmentation. Sci Adv 6(12):eaaz0330

Hortelão AC, Patiño T, Perez-Jiménez A, Blanco À, Sánchez S (2018) Enzyme-powered nanobots enhance anticancer drug delivery. Adv Funct Mater 28(25):1705086

Hortelão AC, Carrascosa R, Murillo-Cremaes N, Patiño T, Sánchez S (2019) Targeting 3D bladder cancer spheroids with ureasepowered nanomotors. ACS Nano 13(1):429-439

Joseph A, Contini C, Cecchin D, Nyberg S, Ruiz-Perez L, Gaitzsch J, Fullstone G, Tian X, Azizi J, Preston J, Volpe G, Battaglia G (2017) Chemotactic synthetic vesicles: design and applications in blood-brain barrier crossing. Sci Adv 3(8):e1700362

Jurado-Sanchez B (2018) Nanoscale biosensors based on selfpropelled objects. Biosensors 8:59

Jurado-Sánchez B, Escarpa A (2017) Janus micromotors for electrochemical sensing and biosensing applications: a review. Electroanalysis 29(1):14-23

Jurado-Sánchez B, Wang J (2018) Micromotors for environmental applications: a review. Environ Sci: Nano 5(7):1530-1544

Jurado-Sánchez B, Pacheco M, Maria-Hormigos R, Escarpa A (2017) Perspectives on Janus micromotors: materials and applications. Appl Mater Today 9:407-418

Khezri B, Pumera M (2019) Metal-organic frameworks based nano/micro/millimeter-sized self-propelled autonomous machines. Adv Mater 31(14):e1806530 
Kiristi M, Singh VV, Esteban-Fernández de Ávila B, Uygun M, Soto F, Aktaş Uygun D, Wang J (2015) Lysozyme-based antibacterial nanomotors. ACS Nano 9(9):9252-9259

Kong L, Guan J, Pumera M (2018) Micro- and nanorobots based sensing and biosensing. Curr Opin Electrochem 10:174-182

Kumar B, Patil AJ, Mann S (2018) Enzyme-powered motility in buoyant organoclay/DNA protocells. Nat Chem 10(11):1154-1163

Li J, Angsantikul P, Liu W, Esteban-Fernánde de Ávila B, Thamphiwatana S, Xu M, Sandraz E, Wang X, Delezuk J, Gao W, Zhang L, Wang J (2017) Micromotors spontaneously neutralize gastric acid for $\mathrm{pH}$-responsive payload release. Angew Chem Int Ed 56(8):2156-2161

Liang J, Liang K (2020) Biocatalytic metal-organic frameworks: prospects beyond bioprotective porous matrices. Adv Funct Mater 30(27):2001648

Liang K, Ricco R, Doherty CM, Styles MJ, Bell S, Kirby N, Mudie S, Haylock D, Hill AJ, Doonan CJ, Falcaro P (2015) Biomimetic mineralization of metal-organic frameworks as protective coatings for biomacromolecules. Nat Commun 6:7240

Liang K, Richardson JJ, Cui J, Caruso F, Doonan CJ, Falcaro P (2016a) Metal-organic framework coatings as cytoprotective exoskeletons for living cells Adv. Mater. 28:7910-7914

Liang K, Ricco R, Doherty CM, Styles MJ, Falcaro P (2016b) Amino acids as biomimetic crystallization agents for the synthesis of ZIF-8 particles CrystEngComm 18:4264-4267

Liang K, Wang R, Boutter M, Doherty CM, Mulet X, Richardson JJ (2017) Biomimetic mineralization of metal-organic frameworks around polysaccharides. Chem Commun 53:1249-1252

Liu M, Sun Y, Wang T, Ye Z, Zhang H, Dong B, Li CY (2016) A biodegradable, all-polymer micromotor for gas sensing applications. J Mater Chem C 4(25):5945-5952

Llopis-Lorente A, García-Fernández A, Murillo-Cremaes N, Hortelão AC, Patiño T, Villalonga R, Sancenón F, Martínez-Máñez R, Sánchez S (2019) Enzyme-powered gated mesoporous silica nanomotors for on-command intracellular payload delivery. ACS Nano 13(10):12171-12183

Ma X, Sánchez S (2017) Bio-catalytic mesoporous Janus nanomotors powered by catalase enzyme. Tetrahedron 73(33):4883-4886

Ma X, Jannasch A, Albrecht U-R, Hahn K, Miguel-López A, Schäffer E, Sánchez S (2015) Enzyme-powered hollow mesoporous Janus nanomotors. Nano Lett 15(10):7043-7050

Ma X, Hortelao AC, Patino T, Sanchez S (2016) Enzyme catalysis to power micro/nanomachines. ACS Nano 10(10):9111-9122

Ma X, Feng H, Liang C, Liu X, Zeng F, Wang Y (2017) Mesoporous silica as micro/nano-carrier: from passive to active cargo delivery, a mini review. J Mater Sci Technol 33(10):1067-1074

Mayorga-Martinez CC, Pumera M (2019) Self-propelled tags for protein detection. Adv Funct Mater 30(6):1906449

Nijemeisland M, Abdelmohsen LKEA, Huck WTS, Wilson DA, van Hest JCM (2016) A compartmentalized out-of-equilibrium enzymatic reaction network for sustained autonomous movement. ACS Central Sci 2(11):843-849

Ning H, Zhang Y, Zhu H, Ingham A, Huang G, Mei Y, Solovev AA (2018) Geometry design, principles and assembly of micromotors. Micromachines (Basel) 9:75

Olson ES, Orozco J, Wu Z, Malone CD, Yi B, Gao W, Eghtedari M, Wang J, Mattrey RF (2013) Toward in vivo detection of hydrogen peroxide with ultrasound molecular imaging. Biomaterials 34(35):8918-8924

Orozco J, García-Gradilla V, D’Agostino M, Gao W, Cortés A, Wang J (2013) Artificial enzyme-powered microfish for water-quality testing. ACS Nano 7(1):818-824
Orozco J, Vilela D, Valdes-Ramirez G, Fedorak Y, Escarpa A, Vazquez-Duhalt R, Wang J (2014) Efficient biocatalytic degradation of pollutants by enzyme-releasing self-propelled motors. Chem Eur J 20(10):2866-2871

Pacheco M, Lopez MA, Jurado-Sanchez B, Escarpa A (2019) Selfpropelled micromachines for analytical sensing: a critical review. Anal Bioanal Chem 411(25):6561-6573

Parmar J, Vilela D, Villa K, Wang J, Sánchez S (2018) Micro- and nanomotors as active environmental microcleaners and sensors. J Am Chem Soc 140(30):9317-9331

Patino T, Feiner-Gracia N, Arque X, Miguel-Lopez A, Jannasch A, Stumpp T, Schaffer E, Albertazzi L, Sanchez S (2018) Influence of enzyme quantity and distribution on the selfpropulsion of non-Janus urease-powered micromotors. J Am Chem Soc 140(25):7896-7903

Patino T, Porchetta A, Jannasch A, Lladó A, Stumpp T, Schäffer E, Ricci F, Sánchez S (2019) Self-sensing enzyme-powered micromotors equipped with pH-responsive DNA nanoswitches. Nano Lett 19(6):3440-3447

Peng F, Tu Y, Wilson DA (2017) Micro/nanomotors towards in vivo application: cell, tissue and biofluid. Chem Soc Rev 46(17):5289-5310

Restrepo-Perez L, Soler L, Martinez-Cisneros C, Sanchez S, Schmidt OG (2014) Biofunctionalized self-propelled micromotors as an alternative on-chip concentrating system. Lab Chip 14(16):2914-2917

Safdar M, Khan SU, Janis J (2018) Progress toward catalytic microand nanomotors for biomedical and environmental applications. Adv Mater 30(24):1703660

Sanchez S, Solovev AA, Mei Y, Schmidt OG (2010) Dynamics of biocatalytic microengines mediated by variable friction control. J Am Chem Soc 132(38):13144-13145

Sattayasamitsathit S, Kaufmann K, Galarnyk M, Vazquez-Duhalt R, Wang J (2014) Dual-enzyme natural motors incorporating decontamination and propulsion capabilities. RSC Adv 4(52):27565-27570

Schattling P, Thingholm B, Städler B (2015) Enhanced diffusion of glucose-fueled Janus particles. Chem Mater 27(21):7412-7418

Schattling PS, Ramos-Docampo MA, Salgueiriño V, Städler B (2017) Double-fueled Janus swimmers with magnetotactic behavior. ACS Nano 11(4):3973-3983

Simmchen J, Baeza A, Ruiz D, Esplandiu MJ, Vallet-Regí M (2012) Asymmetric hybrid silica nanomotors for capture and cargo transport: towards a novel motion-based DNA sensor. Small 8(13):2053-2059

Somasundar A, Ghosh S, Mohajerani F, Massenburg LN, Yang T, Cremer PS, Velegol D, Sen A (2019) Positive and negative chemotaxis of enzyme-coated liposome motors. Nat Nanotechnol 14:1129-1134

Sugai N, Morita Y, Komatsu T (2019) Nonbubble-propelled biodegradable microtube motors consisting only of protein. Chem Asian J 14(17):2953-2957

Sun Y, Ding M, Zeng X, Xiao Y, Wu H, Zhou H, Ding B, Qu C, Hou W, Er-bu AGA, Zhang Y, Cheng Z, Hong X (2017) Novel brightemission small-molecule NIR-II fluorophores for in vivo tumor imaging and image-guided surgery. Chem Sci 8(5):3489-3493

Sun J, Mathesh M, Li W, Wilson DA (2019) Enzyme-powered nanomotors with controlled size for biomedical applications. ACS Nano 13(9):10191-10200

Tang S, Zhang F, Gong H, Wei F, Zhuang J, Karshalev E, EstebanFernández de Ávila B, Huang C, Zhou Z, Li Z, Yin L, Dong H, Fang RH, Zhang X, Zhang L, Wang J (2020) Enzyme-powered Janus platelet cell robots for active and targeted drug delivery. Sci Robot 5(43):6137 
Thomas N, Thornhill RA (1998) The physics of biological molecular motors. J Phys D Appl Phys 31(3):253-266

Tu Y, Peng F, Wilson DA (2017) Motion manipulation of micro- and nanomotors. Adv Mater 29:1701970

Vilela D, Parmar J, Zeng Y, Zhao Y, Sanchez S (2016) Graphenebased microbots for toxic heavy metal removal and recovery from water. Nano Lett 16(4):2860-2866

Wang H, Pumera M (2017) Emerging materials for the fabrication of micro/nanomotors. Nanoscale 9(6):2109-2116

Wang Y, Hernandez RM, Bartlett DJ Jr, Bingham JM, Kline TR, Sen A, Mallouk TE (2006) Bipolar electrochemical mechanism for the propulsion of catalytic nanomotors in hydrogen peroxide solutions. Langmuir 22(25):10451-10456

Wang J, Hu Z, Xu J, Zhao Y (2014) Therapeutic applications of lowtoxicity spherical nanocarbon materials. NPG Asia Mater 6(2):e84-e84

Wang H, Moo JG, Pumera M (2016) From nanomotors to micromotors: the influence of the size of an autonomous bubble-propelled device upon its motion. ACS Nano 10(5):5041-5050

Wang C, Wang Z, Zhao T, Li Y, Huang G, Sumer BD, Gao J (2018) Optical molecular imaging for tumor detection and imageguided surgery. Biomaterials 157:62-75

Wang J, Dong R, Wu H, Cai Y, Ren B (2019a) A review on artificial micro/nanomotors for cancer-targeted delivery, diagnosis, and therapy. Nano-Micro Lett 12(1):11

Wang S, Liu X, Wang Y, Xu D, Liang C, Guo J, Ma X (2019b) Biocompatibility of artificial micro/nanomotors for use in biomedicine. Nanoscale 11(30):14099-14112

Wong F, Dey KK, Sen A (2016) Synthetic micro/nanomotors and pumps: fabrication and applications. Annu Rev Mater Res 46(1):407-432
Wong JKH, Tan HK, Lau SY, Yap P-S, Danquah MK (2019) Potential and challenges of enzyme incorporated nanotechnology in dye wastewater treatment: A review. J Environ Chem Eng $7(4): 103261$

Wu Y, Lin X, Wu Z, Möhwald H, He Q (2014) Self-propelled polymer multilayer janus capsules for effective drug delivery and light-triggered release. ACS Appl Mater Interfaces 6(13):10476-10481

Wu Z, Lin X, Zou X, Sun J, He Q (2015) Biodegradable proteinbased rockets for drug transportation and light-triggered release. ACS Appl Mater Interfaces 7(1):250-255

Ying Y, Pourrahimi AM, Sofer Z, Matejkova S, Pumera M (2019) Radioactive uranium preconcentration via self-propelled autonomous microrobots based on metal-organic frameworks. ACS Nano 13:11477-11487

You Y, Xu D, Pan X, Ma X (2019) Self-propelled enzymatic nanomotors for enhancing synergetic photodynamic and starvation therapy by self-accelerated cascade reactions. Appl Mater Today 16:508-517

Yu X, Cheng H, Zhang M, Zhao Y, Qu L, Shi G (2017) Graphenebased smart materials. Nat Rev Mater 2(9):17046

Zhang X, Chen C, Wu J, Ju H (2019) Bubble-propelled jellyfish-like micromotors for DNA sensing. ACS Appl Mater Interfaces 11(14):13581-13588

Zhao X, Gentile K, Mohajerani F, Sen A (2018) Powering motion with enzymes. Acc Chem Res 51(10):2373-2381

Zhao L, Liu Y, Xie S, Ran P, Wei J, Liu Q Li X (2020) Janus micromotors for motion-capture-ratiometric fluorescence detection of circulating tumor cells. Chem Eng J 382:123041 